\title{
Numerical Investigation on the Effects of Varying the Arc length of a Windshield on the Performance of a Highway Installed Banki Wind Turbine
}

\author{
Ian Jay T. Matias ${ }^{1,2}$, , Louis Angelo M. Danao ${ }^{1, *(\mathbb{D})}$ and Binoe E. Abuan ${ }^{1}$ \\ 1 Department of Mechanical Engineering, University of the Philippines Diliman, \\ Quezon City 1100, Philippines; ianjay.matias@bulsu.edu.ph (I.J.T.M.); beabuan@up.edu.ph (B.E.A.) \\ 2 Department of Mechanical Engineering, Bulacan State University, Bulacan 3017, Philippines \\ * Correspondence: louisdanao@up.edu.ph
}

Citation: Matias, I.J.T.; Danao, L.A.M.; Abuan, B.E. Numerical Investigation on the Effects of Varying the Arc length of a Windshield on the Performance of a Highway Installed Banki Wind Turbine. Fluids 2021, 6, 285. https:// doi.org/10.3390/fluids6080285

Academic Editor: Eldad Avital

Received: 30 June 2021

Accepted: 11 August 2021

Published: 16 August 2021

Publisher's Note: MDPI stays neutral with regard to jurisdictional claims in published maps and institutional affiliations.

Copyright: (c) 2021 by the authors. Licensee MDPI, Basel, Switzerland. This article is an open access article distributed under the terms and conditions of the Creative Commons Attribution (CC BY) license (https:// creativecommons.org/licenses/by/ $4.0 /)$.

\begin{abstract}
Two-dimensional computational fluid dynamics (CFD) simulations are employed to investigate the effects of adding a circular-arc-shaped windshield on the performance of a Banki type vertical axis wind turbine (VAWT), particularly to the application where the VAWT is harnessing wind energy in highway caused by the passing vehicles. Unsteady Reynolds-Averaged Navier-Stokes (URANS) is the computational approach used to calculate the turbulent flow within the domain. Two sets of simulation cases based on two different vehicles (i.e., car and a bus) are performed with varying arc-length of the windshield. The results show that the windshield provides an increase in the energy captured by the VAWT by up to $16.14 \%$ compared to no windshield case when the car model is used. In contrast, windshield in all the simulation cases using a bus model gives a negative effect to VAWT performance where the worst case yields $-64.77 \%$.
\end{abstract}

Keywords: vertical axis wind turbine; Banki wind turbine; highway wind

\section{Introduction}

The complete global transition to renewable energy is still not expected to be achieved in the next few years even in the case of theoretical energy modelling frameworks such as in REMap case [1]. Researchers see that there must be two important steps to take to enable the complete energy transition. First, for those applications where technology solutions exist, the important next step is to establish enabling frameworks that are needed to scale up their deployment. Secondly, for applications where solutions are either at their early stages of commercialization or do not exist at present, the next step is to foster technology innovation, along with enabling policy, and social and financial measures, to rapidly bring the emerging clean technologies to the marketplace [2]. Relating the latter to the sector of wind energy, it is seen that it is important to also give research attention to non-conventional installations of wind turbines such as their application in harnessing energy from the wake of passing vehicles along the highway. Previous studies conducted measurements of wind speed along the highway using hot-wire anemometers installed in specified lateral distances from the road, elevation from the ground, and anemometer orientation [3]. The measurements differed with each varying position and orientation which suggests the unsteady and turbulent behavior of the wake. Among the studies that conducted similar field measurements [3-5], the wind magnitude of the vehicle wake was roughly within the range of 2 to $6 \mathrm{~m} / \mathrm{s}$. The vertical axis wind turbine (VAWT) fits in this application because it does not require a yaw mechanism, allowing it to operate with the wind coming from different directions and it can self-start at a low wind speed. A study conducted performance evaluation of highway VAWT with different rotor type using numerical simulations where the Banki rotor bested Savonius and Darrieus [6]. It was found out that the lift type Darrieus VAWT can hardly produce power due to very highly 
turbulent and complex wind behavior, therefore, it is not suitable for highway turbine application. Banki and Savonius being drag-type VAWTs gave better results, however, Savonius has a lower performance due to the close-center design causing a greater amount of block effect on the flow.

Despite a number of studies showing the capability of VAWT to harness wind energy from vehicle wakes $[4,7,8]$, the general acceptability of such system remains low, as there is still no large installation to date. This calls for ideas to improve the performance of the system so it can be more attractive. Several studies investigated the effect of applying augmentation devices that block and divert incoming steady wind to the VAWT and obtained good results [9-15]. This motivates the present study to apply a similar concept in highway VAWT where wind input is from vehicle wake instead of a steady wind source. The chosen augmentation device is a windshield similar to that in [11] which is intended to block the portion of incoming wind that go against the VAWT rotation causing the reduction of torque. The effect of the windshield with different arclengths is investigated by subjecting the flow domain into CFD simulations with a two-dimensional, incompressible URANS as the approach. Its effectiveness is checked using two vehicle types, one a car model and the other a bus.

\section{Materials and Methods}

Two-dimensional geometries are used to model the vehicles, VAWT, and the windshield. The VAWT is assumed to be positioned beside the highway dedicated to harvesting wind energy from passing vehicles in one lane only, for this study. Further, installation in the median of the highway may not be possible due to an already constructed road where it can be too narrow or too wide to fit the operation envelope of the VAWT. The study is focused on the effect of the windshield and is limited on simulating a vehicle passing the VAWT proximity; the effects of other factors like the presence of obstacles (e.g., traffic barriers, posts, trees, etc.) and ambient wind are not included.

\subsection{Geometries and Boundary Conditions}

Car and bus models are used and separately simulated. Both vehicles are run at a constant speed of $28 \mathrm{~m} / \mathrm{s}$ (approx. $100 \mathrm{kph}$ ) where the initial position is at $84 \mathrm{~m}$ from the VAWT center or equivalent to $3 \mathrm{~s}$ before reaching the VAWT. The vehicle translates parallel to the horizontal axis that is intersecting the VAWT center. The gap between the edge of the vehicle and the VAWT edge is $1 \mathrm{~m}$. Figure 1 illustrates the above description.

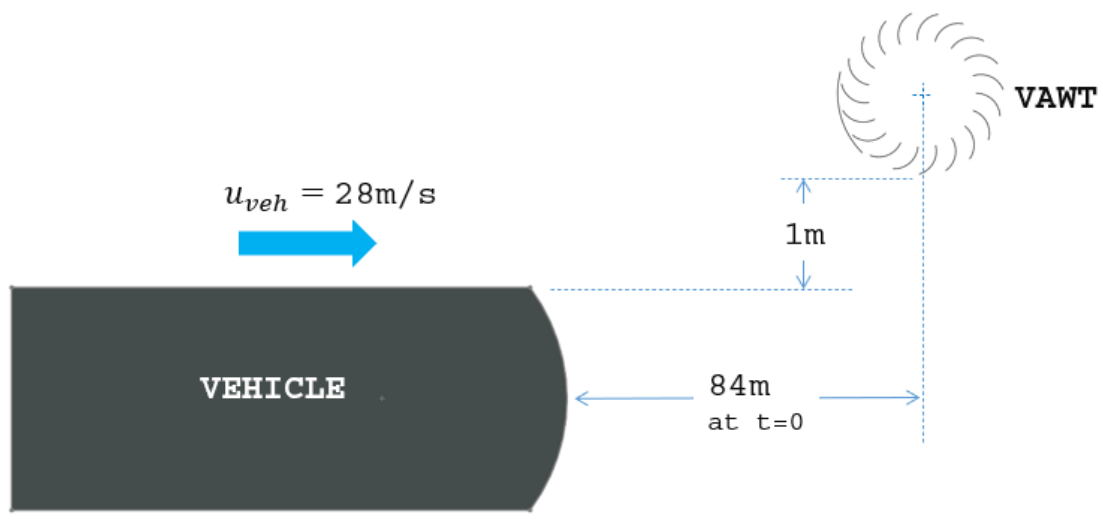

Figure 1. Coordinates of the vehicle and VAWT.

The 2D geometries for the vehicles and Banki wind turbine are shown in Figures 2 and 3, respectively. The car is $1.8 \mathrm{~m}$ wide and $4.5 \mathrm{~m}$ long, with a squared back and circular arc in front with a radius of $0.9 \mathrm{~m}$ [7], while the bus width, length, and circular arc radius are $2.4 \mathrm{~m}, 8 \mathrm{~m}$, and $2 \mathrm{~m}$, respectively [8]. The selected Banki wind turbine has 20 equally spaced blades with inner and outer blade circle diameters at $330 \mathrm{~m}$ and $500 \mathrm{~m}$, respectively. 
The blade radius is $146.8 \mathrm{~mm}$ with a blade center at $361.2 \mathrm{~mm}$ from the rotor center [7]. Lastly, the windshield is a circular arc with a thickness of $5 \mathrm{~mm}$ and a distance of $15 \mathrm{~mm}$ from the rotor circumference, see Figure 4 . The windshield arc length is measured by its central angle $(\theta)$ and varies as per the simulation cases. The initial side, which is common for all simulation cases of the same vehicle model, is to be determined based on the dominant wind direction of the wake of that vehicle model. The dominant wind direction is determined by simulating the vehicle passing the VAWT area and applying velocity monitors to obtain the instantaneous wind direction and magnitude averaged within this area. Note that in this simulation, the VAWT blades are removed so it will not influence the vehicle wake. The velocity magnitude of the wake is used as the weight factor to get the weighted-average wind direction. Upon obtaining this, the initial side of the windshield central angle will be positioned against the wind direction.
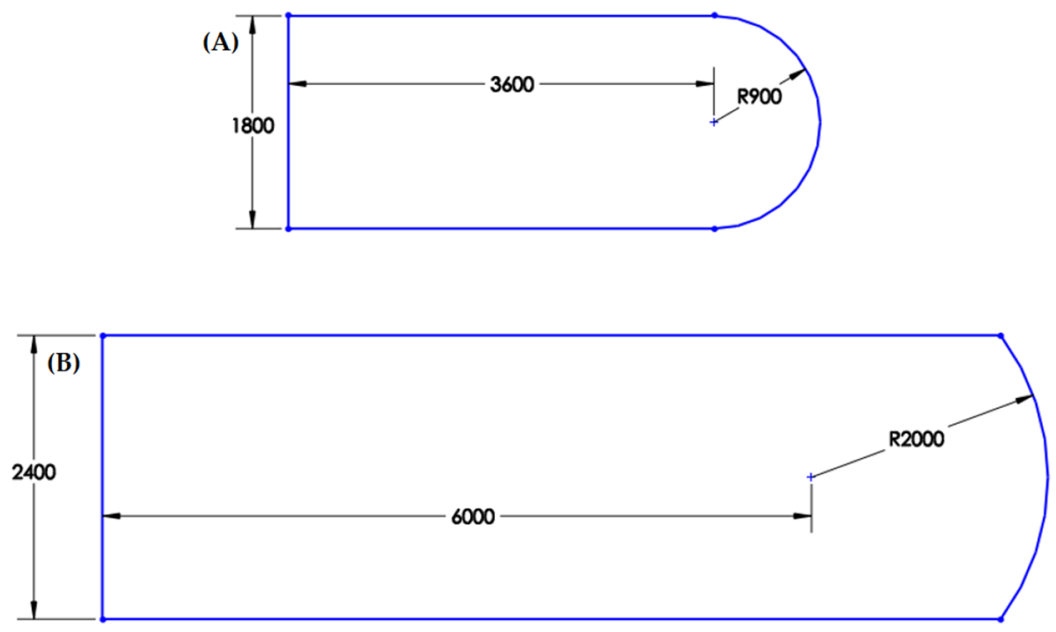

Figure 2. Vehicle geometries: car (A) and bus (B).

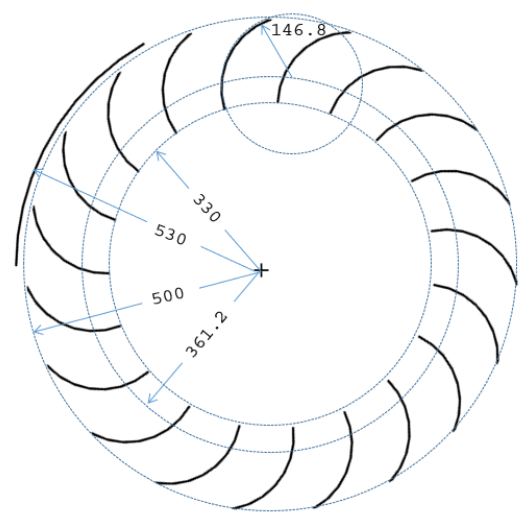

Figure 3. Dimensions of Banki wind turbine with 20 blades, units in mm.

The computational domain is consisting of four kinds of zones: the rotating zone, layering zone, translating zone, and stationary zone. The rotating zone is a circle containing the rotor. The translating zone is where the vehicle is located, this zone moves within the layering zone. The layering zone is the areas in front and tailing the translating zone stretched to near the $+x$ and $-x$ boundaries. Lastly, the stationary zone is the rectangle enclosing all other zones. The dimensions of the zones are specified in Figure 5. The length and width of the entire domain are initially set to $280 \mathrm{~m} \times 70 \mathrm{~m}$. Velocity contour on initial simulations is checked and there must be a negligible variation near the domain outer boundaries to conclude that this domain size is sufficient. 


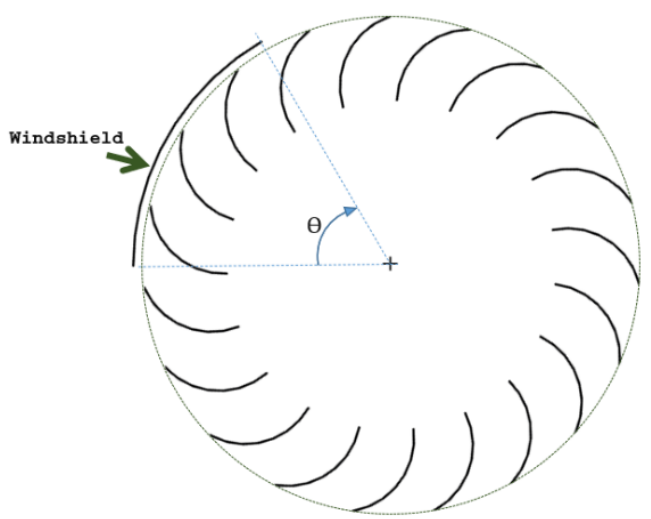

Figure 4. Windshield arc length, $\theta$.

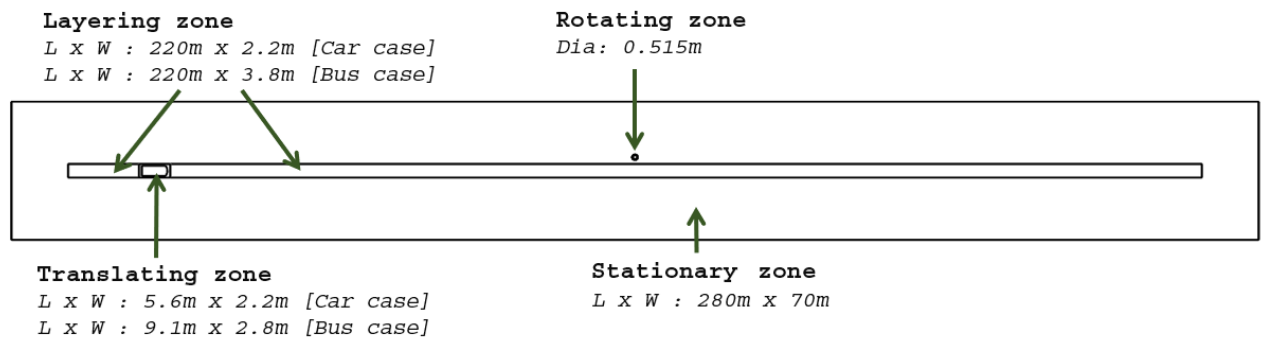

Figure 5. Dimension of cell zones.

The pressure outlet is applied in the left and right boundaries of the stationary zone with backflow direction normal to the boundary, while for the top and bottom, the symmetry boundary condition is used to serve as a 'slip-wall'. The overlapping edges of the zones are set as Interface boundaries to allow transport of flow properties. The left and right edges of the translating zone are set as Interiors, these will translate together with the vehicle walls. Wall with the no-slip condition is imposed on the edges of turbine blades, windshields, and the vehicles. The above description is illustrated in Figure 6.

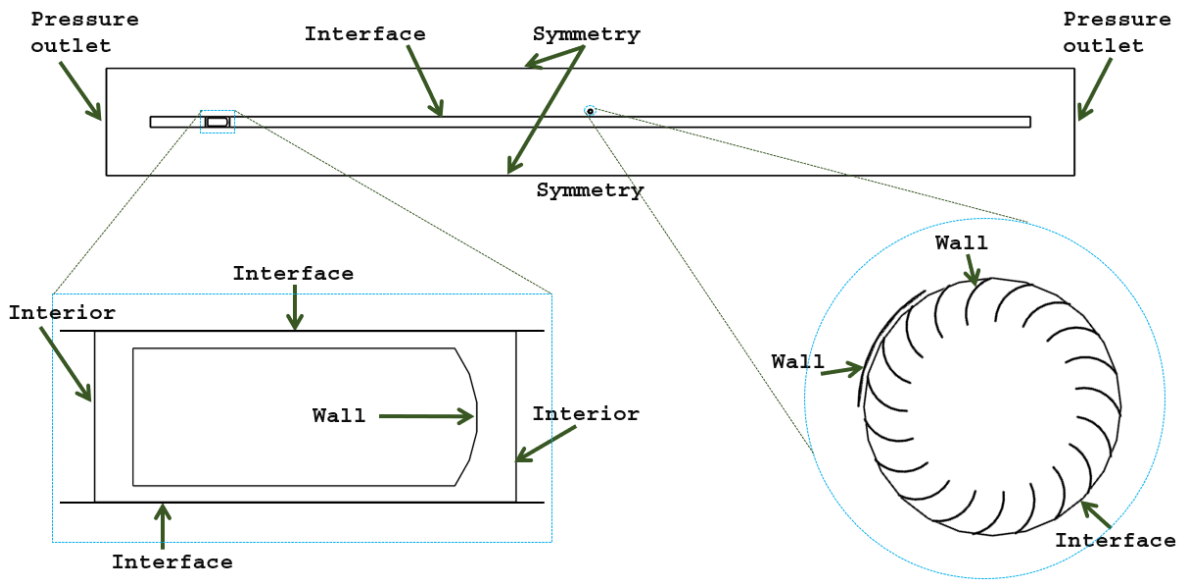

Figure 6. Boundary conditions and mesh interface.

Setting the appropriate cell conditions for the different zones is necessary to realize the simulation cases. In the translating zone, wall and mesh motions equal to vehicle velocity is imposed. For the layering zone, dynamic mesh with the layering method is applied. This allows the deletion of immediate cells in front of the passing translating zone and the creation of new cells immediately behind it. The dynamic mesh method is also applied in 
the rotating zone to realize rotation caused by the forces in the wake of the passing vehicle. Methods to rebuild the mesh are not applied in this zone since the entire zone is rotating, hence, the cells within it remain unchanged.

\subsection{Mesh Generation and Solution Set-Up}

The mesh is created in such a way that there is an increasing resolution towards the region where fluid motion is more dynamic, as shown in Figure 7 . The structured mesh is used as much as possible, however, it is hard to apply on zones that have curves like in the translating and rotating zones without resulting in poor cell skewness and aspect ratio. That is why unstructured mesh is used in these zones. Also, the unstructured mesh is used in surrounding the layering zone to allow transition of the fine structured mesh, surrounding the path of the vehicle, to the coarse structured mesh in the rest of the stationary zone. The first cell height in all walls is set according to the desired wall y-plus values of approximately equal to 1 which is the requirement of the chosen turbulence model, $k-\omega$ SST. In determining the number of elements to be used, a mesh convergence study is performed as detailed in Section 3.1.

The pressure-based coupled computational fluid dynamics (CFD) in ANSYS Fluent 19.2 (ANSYS Inc., Canonsburg, PA, USA) is used with second-order implicit for transient formulation and second-order spatial discretization of pressure, momentum, $k$, and $\omega$ for more accurate computation. The time-step is determined based on the result of the mesh convergence study in Section 3.1, in which the time-step is to be small enough not to exceed the cell height in the layering zone. For instance, if the cell height in layering zone is $60 \mathrm{~mm}$, the time-step must be less than 2.14 milliseconds. Otherwise, the translating zone that is moving at $28 \mathrm{~m} / \mathrm{s}$ will skip the cells in the layering zone that will cause the calculation to halt due to error.

\subsection{Simulation Cases}

Pre-requisite to the simulation cases that are necessary for the parametric study are the simulations for (a) obtaining the coefficient of power $\left(C_{P}\right)$ versus tip-speed ratio (TSR) curve for the VAWT, necessary for comparing the performance of VAWT when it is free to rotate with wind and when only inertia is considered, and (b) knowing the dominant wind directions of the car and bus wakes which are necessary for positioning the windshield.

For (a), the coefficient of power is calculated as per Equation (1):

$$
C_{P}=\frac{P}{0.5 \rho u_{\infty}^{3} S}
$$

where $P$ is the power generated, $u_{\infty}$ is the freestream velocity which will be determined using initial simulation (b) and $S$ is the reference area of the VAWT which is equal to the product of its height and diameter.

For (b), the dominant wind direction is represented by the angle $B$ which is calculated as per Equation (2):

$$
B=\frac{\sum_{1}^{n} u_{i} \beta_{i}}{\sum_{1}^{n} u_{i}}
$$

The $u_{i}$ is the instantaneous velocity magnitude and $\beta_{i}$ is the instantaneous velocity direction, both are averaged values within the rotor zone for each time-step. The $n$ is the number of data points. The angles $B$ and $\beta_{i}$ are both measured from $+x$ axis when a local coordinate is set with origin at the VAWT center, see Figure 8. 


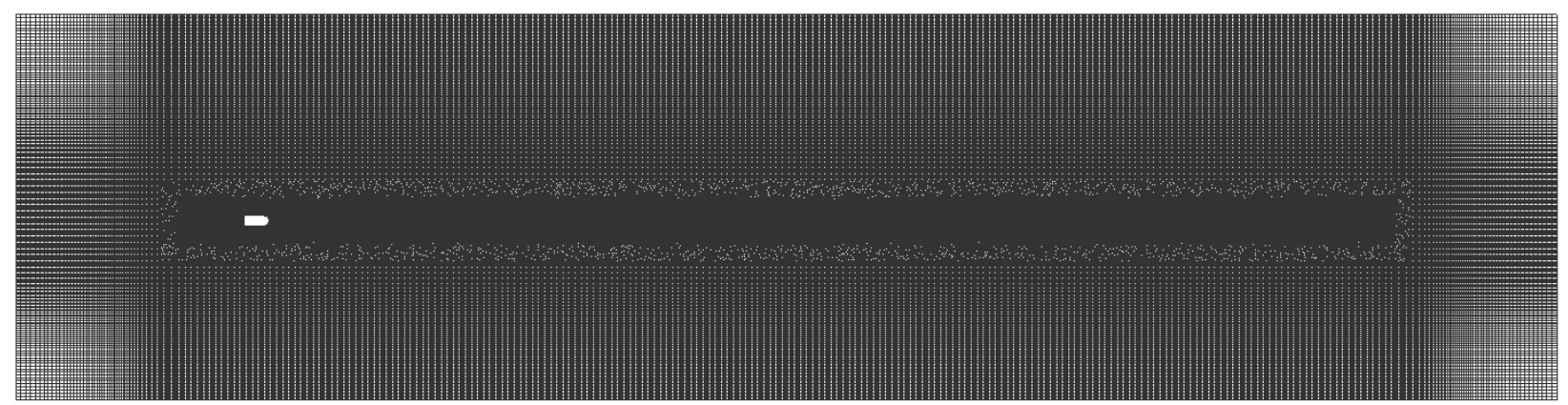

(a)

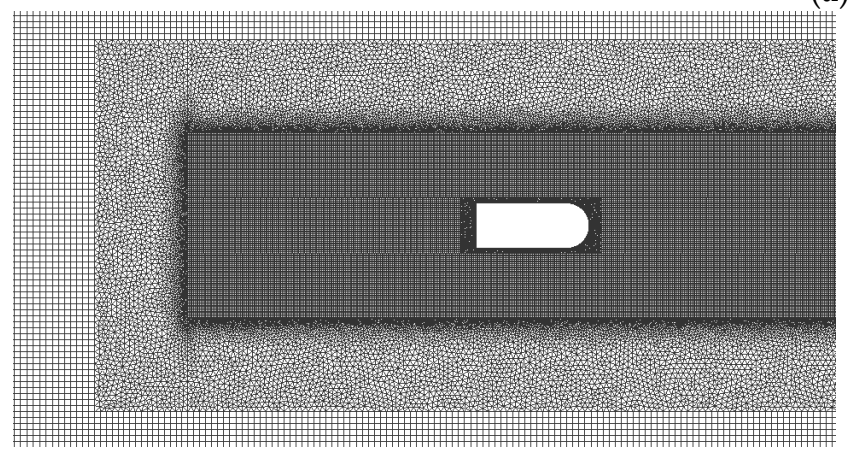

(b)

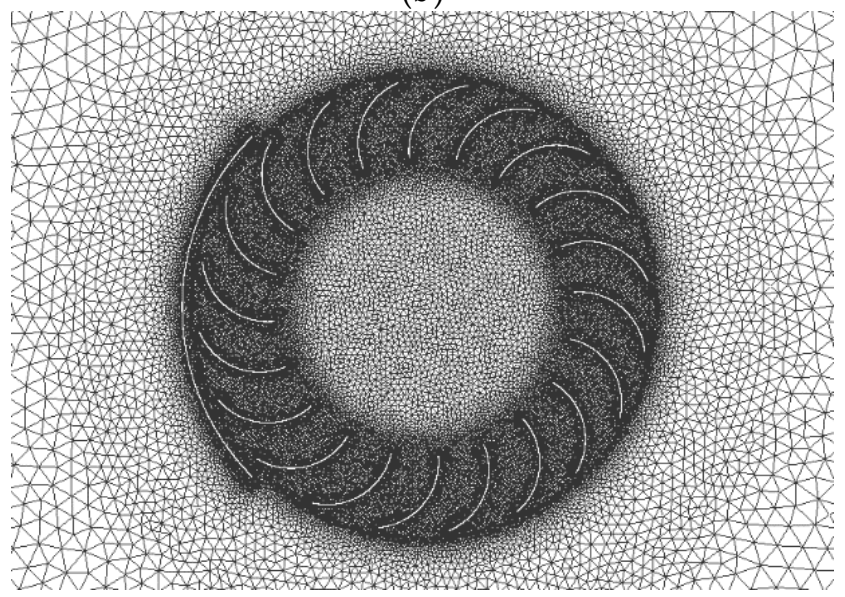

(d)

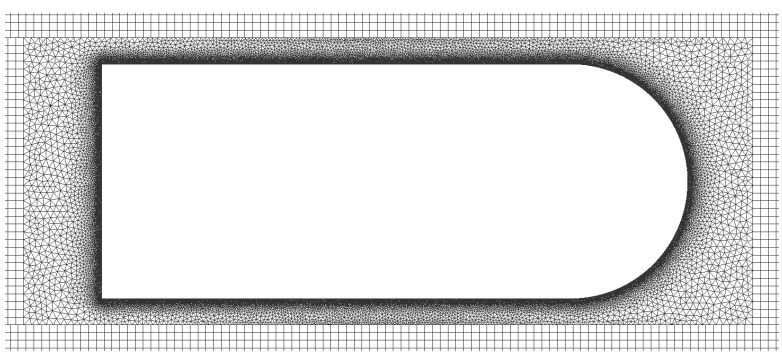

(c)

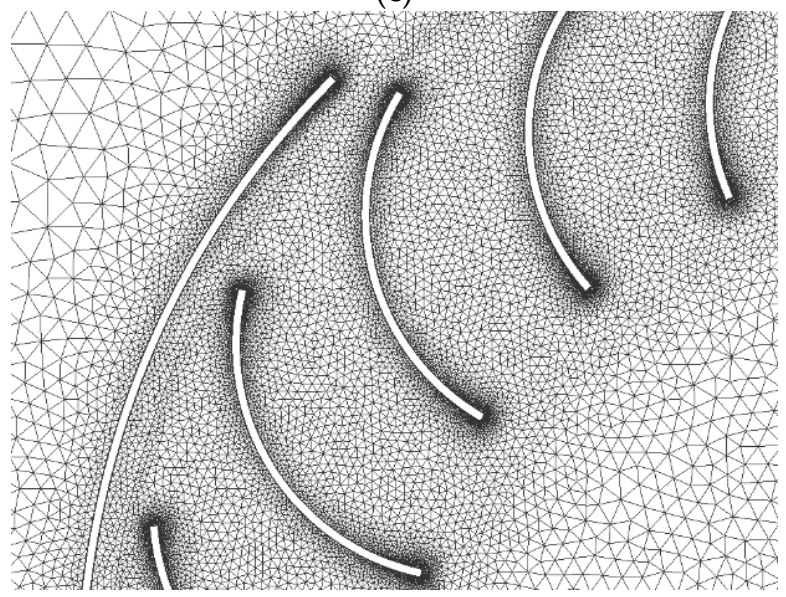

(e)

Figure 7. The mesh: (a) entire computational domain; (b) unstructured mesh surrounding layering zone; (c) translating zone; (d) rotor zone; (e) near blades and windshield.

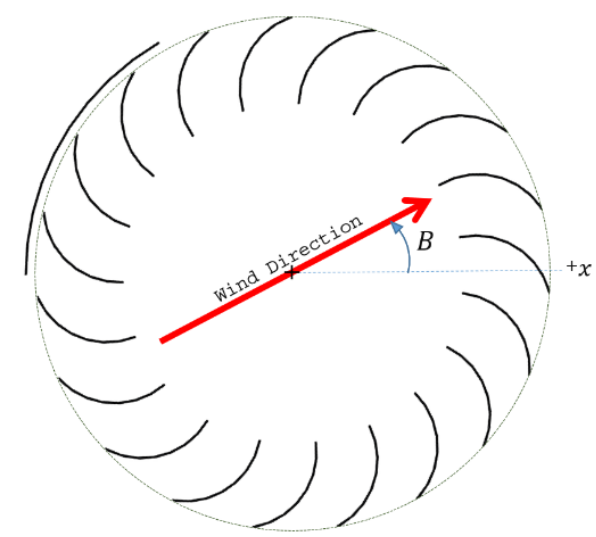

Figure 8. Reference for angles $B$ and $\beta_{i}$. 
There are 6 simulations for Car Case (CC) and another 6 simulations for Bus Case (BC) for the evaluation of the effect of adding the windshield and varying its arc length to the performance of Banki wind turbine. Table 1 shows a summary of the simulation cases.

Table 1. Simulation cases for car case and bus case.

\begin{tabular}{cc}
\hline Car Case/Bus Case & Arc Length $\left(^{\circ}\right)$ \\
\hline 1 & 0 \\
2 & 30 \\
3 & 60 \\
4 & 90 \\
5 & 120 \\
6 & 150 \\
\hline
\end{tabular}

\subsection{VAWT Performance}

The evaluation of VAWT performance for each case is determined by the amount of energy $(E)$ that the VAWT can produce from one pass of a vehicle. This quantity, expressed in Watt-sec, is obtained by taking the area under the instantaneous power $\left(P_{\text {ins }}\right)$ versus time $(t)$ curve using trapezoidal rule in Equation (3) while disregarding the negative value which has no real meaning. For instantaneous power $\left(P_{\text {ins }}\right)$, it is obtained by multiplying the instantaneous torque $\left(T_{\text {ins }}\right)$ and instantaneous rotor velocity $\left(\omega_{\text {ins }}\right)$, as in Equation (4):

$$
\begin{gathered}
E=\sum_{0}^{n-1} \frac{1}{2}\left(P_{i n s_{i+1}}+P_{i n s_{i}}\right)\left(t_{i+1}-t_{i}\right) \\
P_{\text {ins }}=T_{\text {ins }} * \omega_{\text {ins }}
\end{gathered}
$$

Both $T_{\text {ins }}$ and $\omega_{\text {ins }}$ are easily obtained by creating monitors in the CFD solver. These quantities are influenced by the rotor inertia $(J)$ which is calculated using Equation (5):

$$
J=\sum_{1}^{k} m_{b l d} r_{i}^{2}
$$

where $m_{b l d}$ is the mass of each blade, $r_{i}$ is the distance of blade centroid from the VAWT center, and $k$ is the number of blades. The mass of the blade is determined by taking the product of the blade cross-section area, the VAWT height which is $1 \mathrm{~m}$, and the blade density which is assumed equal to steel density which is $8030 \mathrm{~kg} / \mathrm{m}^{3}$.

As part of the limitation of this study, the resistive torques such as the cogging torque are not considered in the calculation of $T_{i n s}$ and $\omega_{i n s}$.

\section{Results \& Discussion}

\subsection{Mesh Convergence and Domain Size Confirmation}

The domain using coarse mesh is initially tested to obtain the instantaneous torque at the rotating VAWT and plotted it versus time. Then, the total number of elements is increased by approximately $20 \%$ and run with the same setup to get the corresponding instantaneous torque curve. The process of successively increasing the mesh elements continues until there is no significant change in instantaneous torque values. At this point, the result is no longer affected by the increasing number of elements, hence the mesh converged. There are three successive mesh densities that are considered as shown in detail in Table 2. 
Table 2. Details of coarse, medium, and fine mesh.

\begin{tabular}{ccccc}
\hline Mesh & $\begin{array}{c}\text { Total Elements, } \\
\text { in Million }\end{array}$ & Construction & $\begin{array}{c}\text { Skewness } \\
\text { (WAVG/Max) }\end{array}$ & $\begin{array}{c}\text { Aspect Ratio } \\
\text { (WAVG/Max) }\end{array}$ \\
\hline \multirow{2}{*}{ Coarse } & 1.33 & Structured & $0.052 / 0.501$ & $1.48 / 3.15$ \\
& \multirow{2}{*}{1.57} & Unstructured & $0.190 / 0.677$ & $1.32 / 2.95$ \\
Medium & \multirow{2}{*}{1.88} & Structured & $0.052 / 0.500$ & $1.35 / 3.20$ \\
& & Unstructured & $0.189 / 0.678$ & $1.32 / 2.96$ \\
Fine & Structured & $0.051 / 0.501$ & $1.34 / 3.02$ \\
& & Unstructured & $0.188 / 0.678$ & $1.32 / 2.99$ \\
\hline
\end{tabular}

Wall $y+$ for all simulations are monitored. Only those simulations with $y+\approx 1$ for all walls are considered acceptable for the evaluation process. The $y+$ monitors are reported using the mean value and the standard deviation for each time-step. For instance, presented below in Figure 9 are the $y+$ monitors taken from car case with medium mesh.

The computer used has 8 physical cores with 64 GB memory. Using all available cores, the simulations took $38 \mathrm{~h}, 46 \mathrm{~h}$, and $57 \mathrm{~h}$ for coarse mesh, medium mesh, and fine mesh, respectively; the resulting torque versus time curve are shown in Figure 10. The coarse mesh is not a good choice due to its inaccuracy, while the 11-h difference between the medium mesh and fine mesh gives significance when considering the number of simulations in the parametric study for the effect of windshields. Therefore, to save time on completing the simulations with a small compromise with the accuracy of the solution, the medium mesh is chosen.

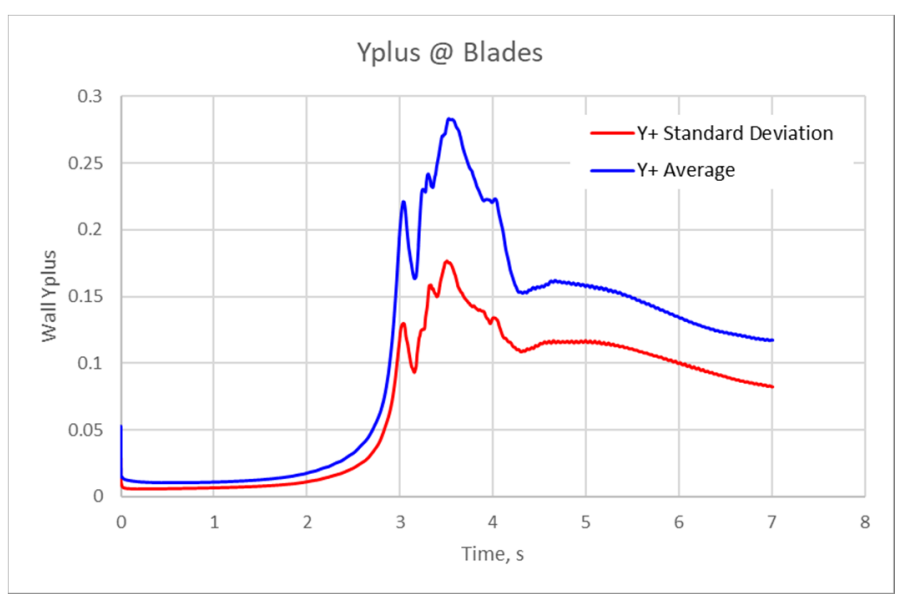

(a)

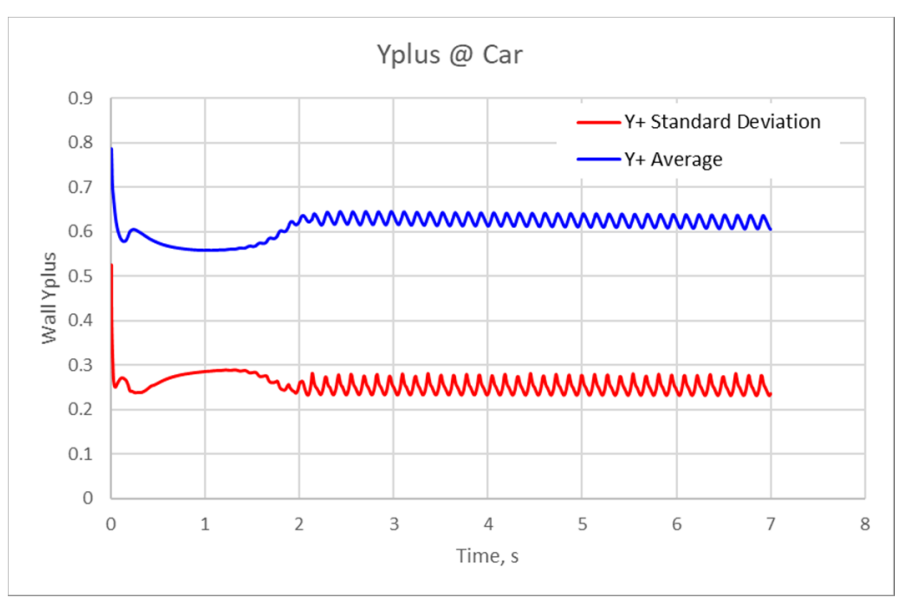

(b)

Figure 9. Wall $y+$ taken from medium mesh (a) at blade walls, (b) at car walls.

For the confirmation of domain size, a velocity contour taken at $t=7 \mathrm{~s}$ using the medium mesh density with the variable range set to only 0 to $5 \mathrm{~m} / \mathrm{s}$, is used and shown in Figure 11. It can be seen that there is no variation in velocity magnitude exceeding $0.4 \mathrm{~m} / \mathrm{s}$ in the far-field. This value is relatively small compared to the magnitude in the path of the vehicle in the VAWT area, which means that the outer boundaries are far enough to affect the area of interest within the domain. Therefore, the initial dimension of $280 \mathrm{~m}$ by $70 \mathrm{~m}$ is confirmed to be suitable for the simulation. 


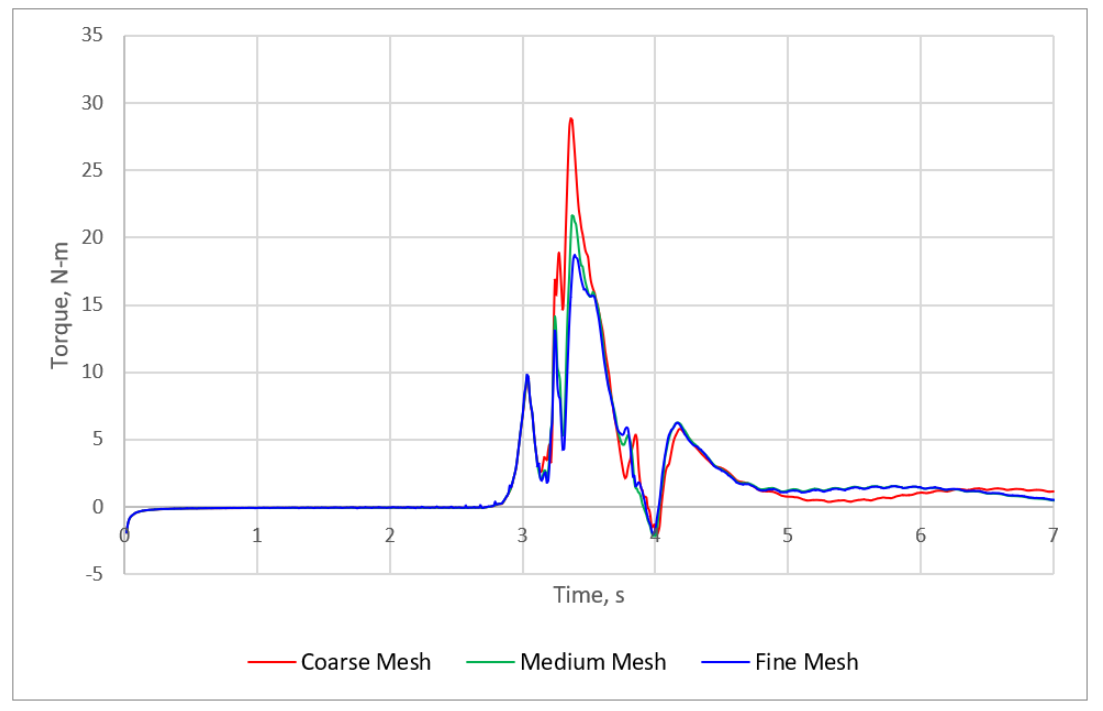

Figure 10. Torque vs. time curve at $\omega=2 \mathrm{rad} / \mathrm{s}$ for difference mesh densities.

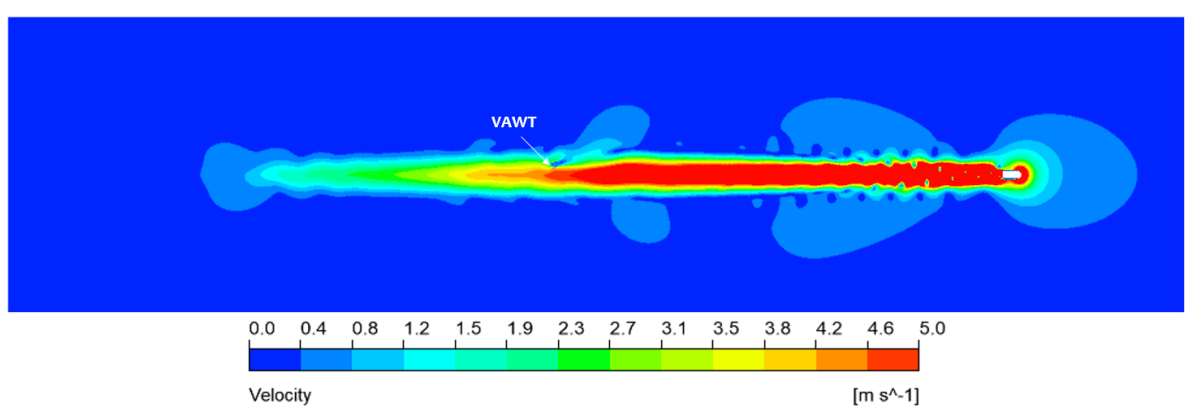

Figure 11. Velocity contour at $t=7 \mathrm{~s}$ using medium mesh density.

\subsection{Wind Profile and Windshield Position}

Knowing the prevailing wind direction that hits the VAWT is the key on proper positioning the windshield so that it would be effective in blocking the incoming wind causing the reduction of torque in the VAWT. From the monitors set, the plots for the instantaneous velocity magnitude and direction are obtained and shown in Figure 12.

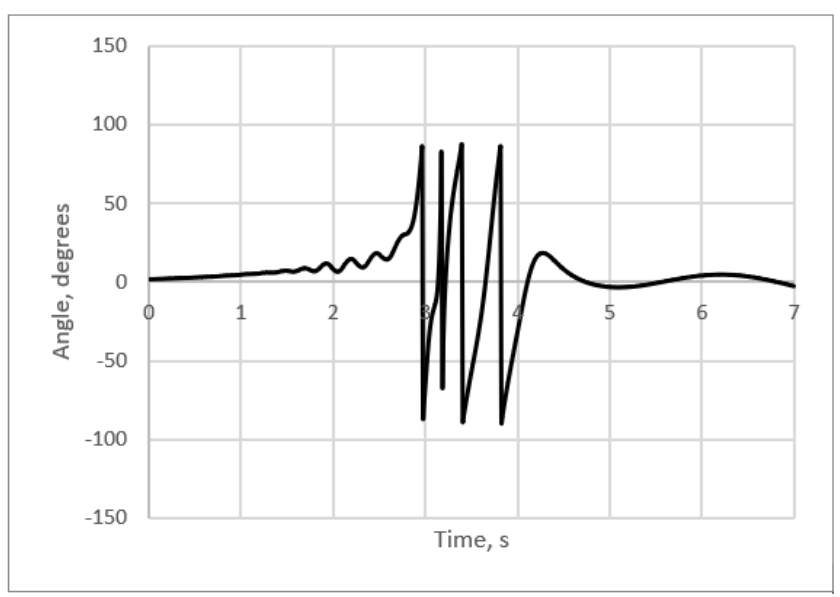

(a)

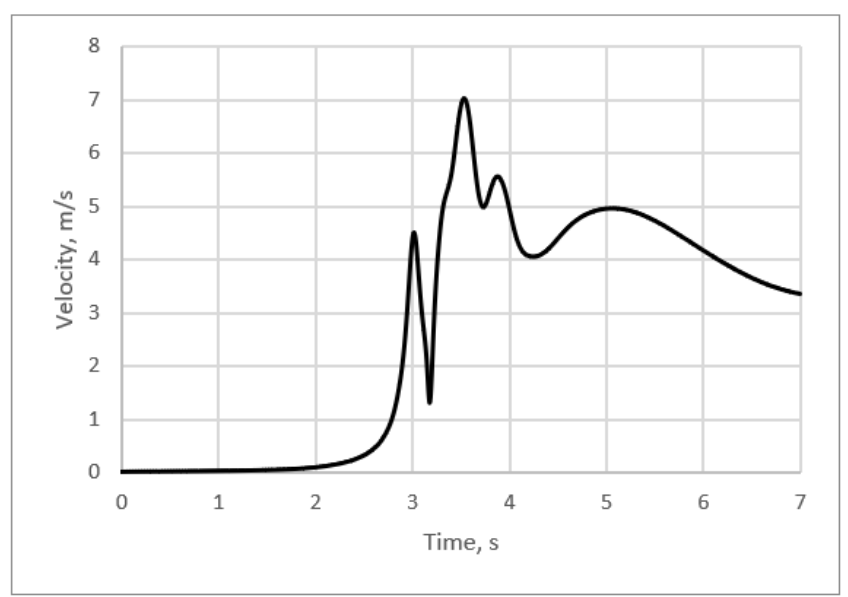

(b)

Figure 12. (a) Instantaneous wind direction at the rotor zone for car model; (b) Instantaneous wind magnitude at the rotor zone for car model. 
The plots show that the wind direction has an increasing slope until $t=3 \mathrm{~s}$ which is the timing to which the vehicle approaches the VAWT location. Then, the wind direction changes rapidly between $t=3 \mathrm{~s}$ to $t=4.5 \mathrm{~s}$ and becomes stable in an almost horizontal direction from $t=4.5 \mathrm{~s}$ and beyond. The behavior of the wind relative to the VAWT as shown in the plots can be visualized in the velocity vector field shown in Figure 13 with the VAWT moving from right to left within the shown domain. The corresponding time on when the specific portion of the wake passes the VAWT, represented by red-outlined circle, is shown in the figure. The figure shows that the rapidly changing wind direction between $t=3 \mathrm{~s}$ and $t=4.5 \mathrm{~s}$ is due to the strong vortices created after the flow separation in the car walls that eventually dissipates in $t>4.5 \mathrm{~s}$ which explains the flattening of curve to almost horizontal direction as seen in the graph.

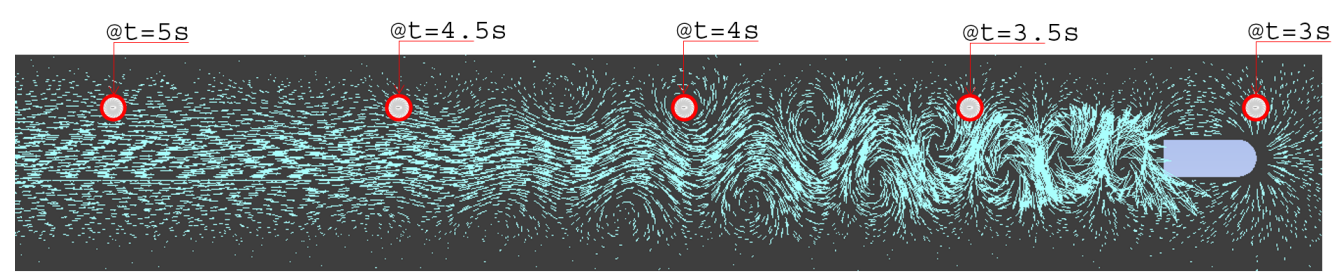

Figure 13. Velocity vector of the car wake taken in stationary zone adjacent to the layering zone.

Since there is no significant magnitude of wind detected before $t=2.5 \mathrm{~s}$, the weightedaverage wind direction is only taken from $t=2.5 \mathrm{~s}$ to $t=7 \mathrm{~s}$ with the weight factor based on its corresponding instantaneous velocity magnitude. Calculating this using Equation (2) gives the value of -1.5 degrees which can be approximated to 0 degree. The windshield must be positioned to block the incoming wind hitting against the rotation of the VAWT; therefore, based on the prevailing wind direction, the windshield is positioned where the starting location is at negative $x$-axis and extends in a clockwise manner.

The same procedure on determining the prevailing wind direction in car case is also done to the bus case. The bus instantaneous wind direction and magnitude are shown in Figure 14 where it can be seen that in contrast with car case, the bus wind direction does not stabilize to any time interval within $t=0 \mathrm{~s}$ to $t=7 \mathrm{~s}$. The same is true when seen in the velocity vector in Figure 15, where it shows that large vortices have not yet dissipated even at $t=4.5 \mathrm{~s}$. The resulted weighted average wind direction for bus is at 15.7 degrees or approximately 16 degrees from positive x-axis. Therefore, for bus case, the windshield is positioned where the starting location is at +16 degrees from negative $x$-axis and extends in a clockwise manner.

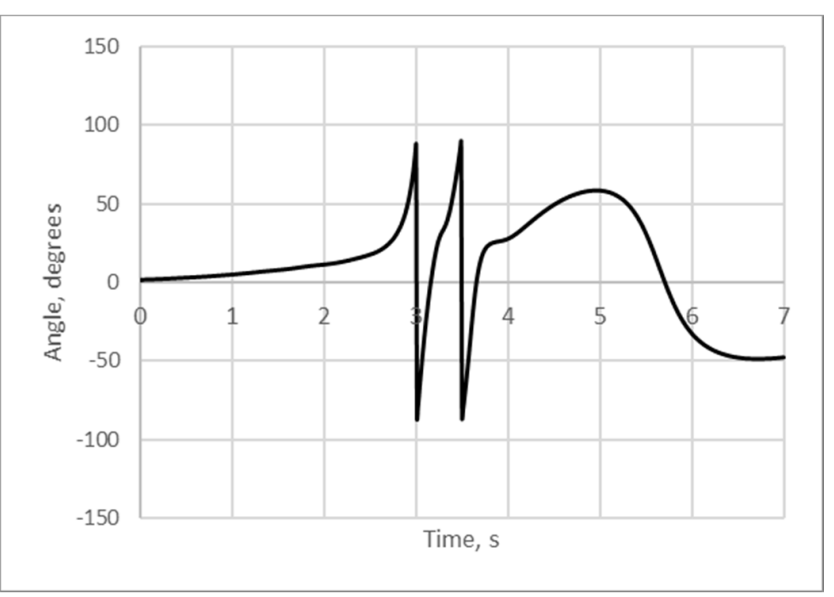

(a)

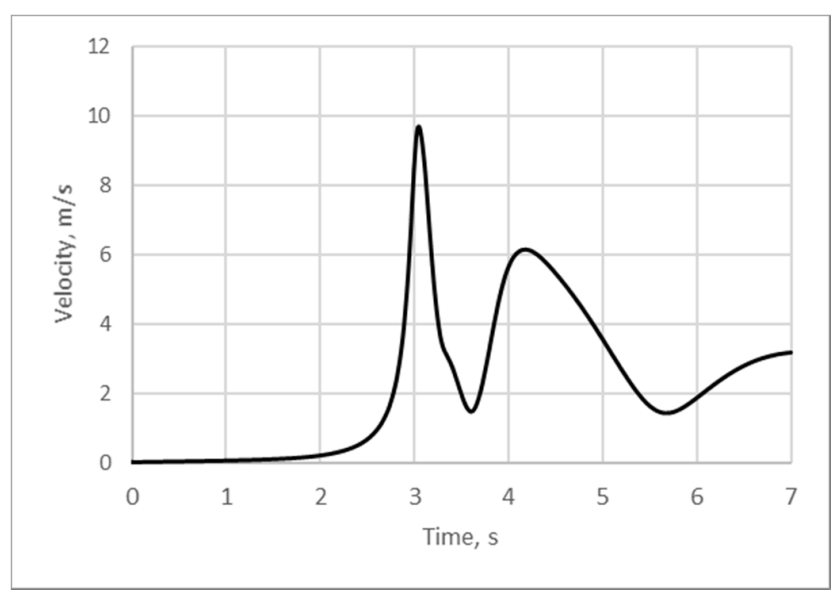

(b)

Figure 14. (a) Instantaneous wind direction at the rotor zone for bus model; (b) Instantaneous wind magnitude at the rotor zone for bus model. 


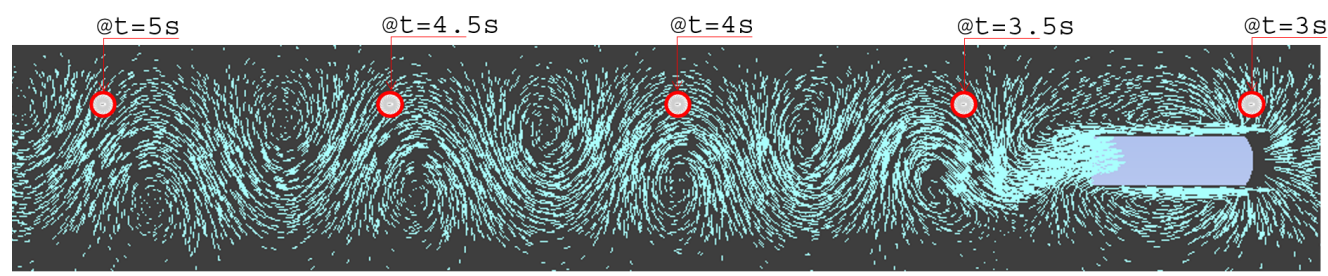

Figure 15. Velocity vector of the bus wake taken in stationary zone adjacent to the layering zone.

\subsection{VAWT $C_{P}$ versus TSR}

A separate domain shown in Figure 16 is prepared for the simulation to compare the $C_{P}$ of VAWT between two conditions; the first is when induced constant rotation is applied, and second when the VAWT is freely rotating with the wind. The same meshing of rotor zone obtained from the mesh convergence study is used for this purpose. The velocity inlet is located at the left half of the domain circumference which is set to $4.4 \mathrm{~m} / \mathrm{s}$. This value is equal to the average velocity taken from the instantaneous values in Figure 12, between $t=2.5 \mathrm{~s}$ to $t=7 \mathrm{~s}$. The results of the simulations for the two aforementioned conditions are plotted together with the $C_{P}$ curve from reference [11], see Figure 17. It can be seen that the $C_{P}$ curve from 5 constant TSR's of the current study is in good agreement with the literature. On the other hand, the instantaneous torque curve that corresponds to a TSR of 0.1 to 0.5 is extracted to arrive with the $C_{P}$ curve for the freely rotating VAWT which is also plotted in Figure 17, see green line. The result of simulation for VAWT without induced rotation are roughly agreeing with the other two $C_{P}$ curves, only that this is based on instantaneous values while the other two are averaged, thus, the main discrepancy. For the rest of the simulations, the VAWT power is calculated in the same manner as the no induced rotation case.

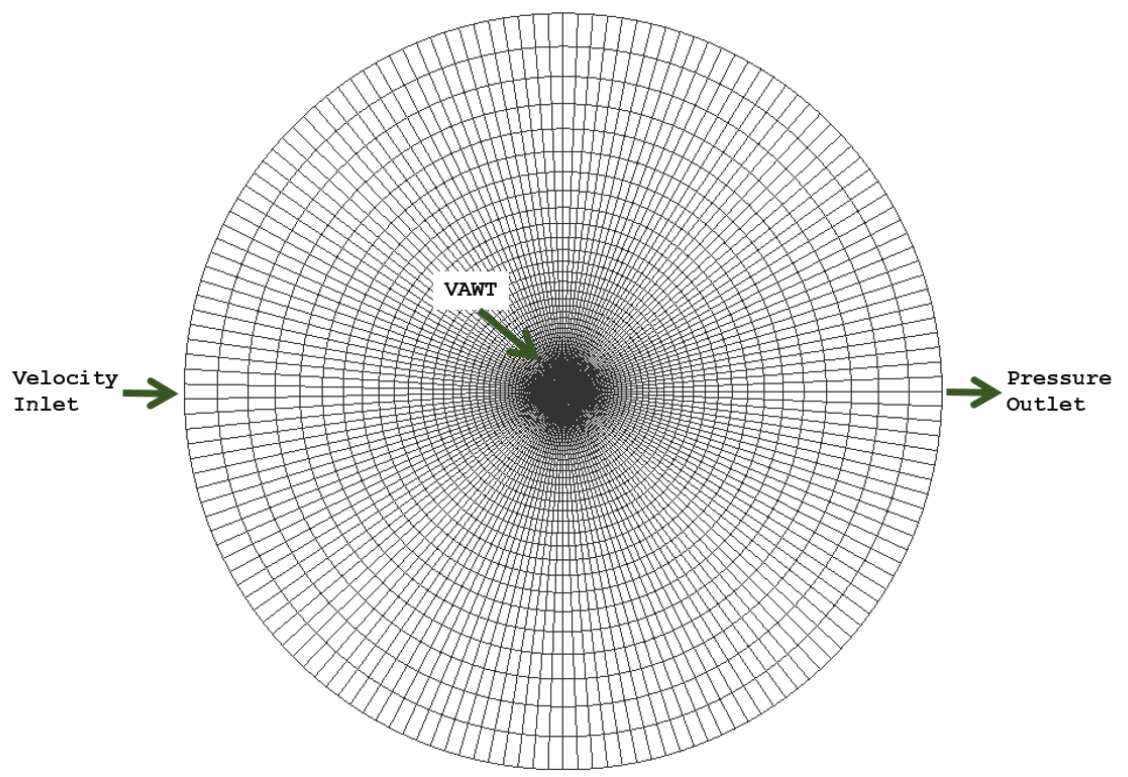

Figure 16. Domain for obtaining the VAWT $C_{P}$ versus TSR curve. 


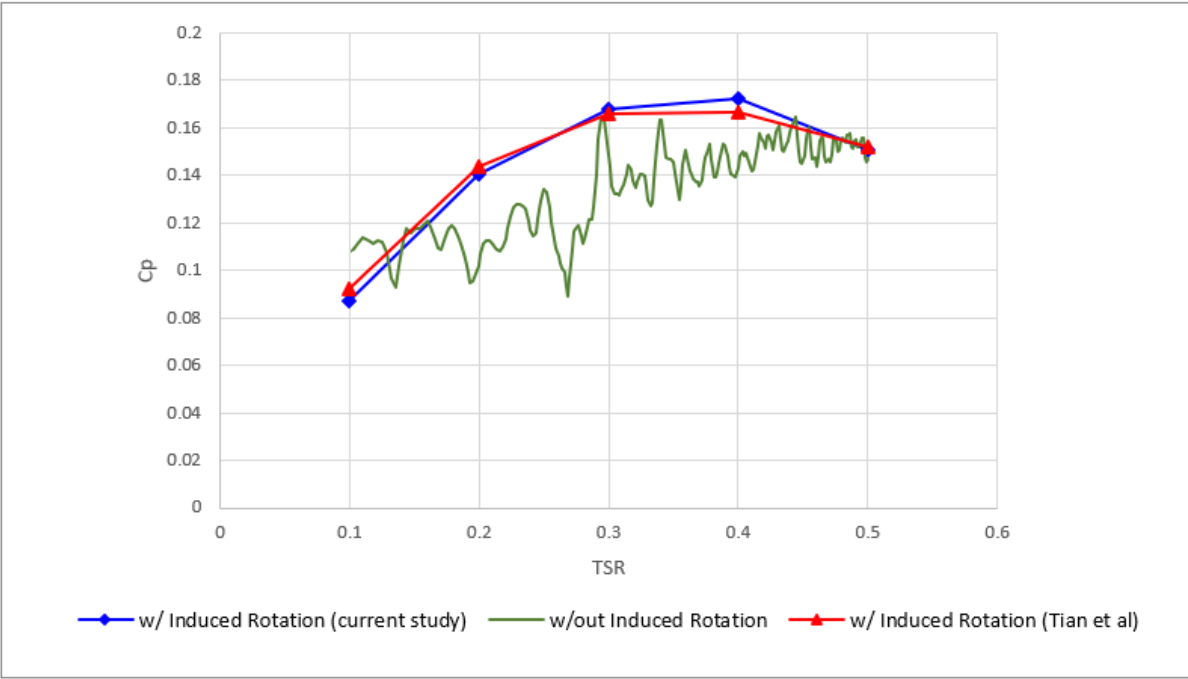

Figure 17. $C_{P}$ versus TSR for VAWT simulated from steady wind of $4.4 \mathrm{~m} / \mathrm{s}$.

\subsection{Effects of Windshield and Vehicle Geometry to VAWT Power}

The instantaneous power curves are obtained using the instantaneous torque and angular velocity from simulation monitors and applying the method stated in Section 2.4. All the car cases are solved and plotted altogether in Figure 18.

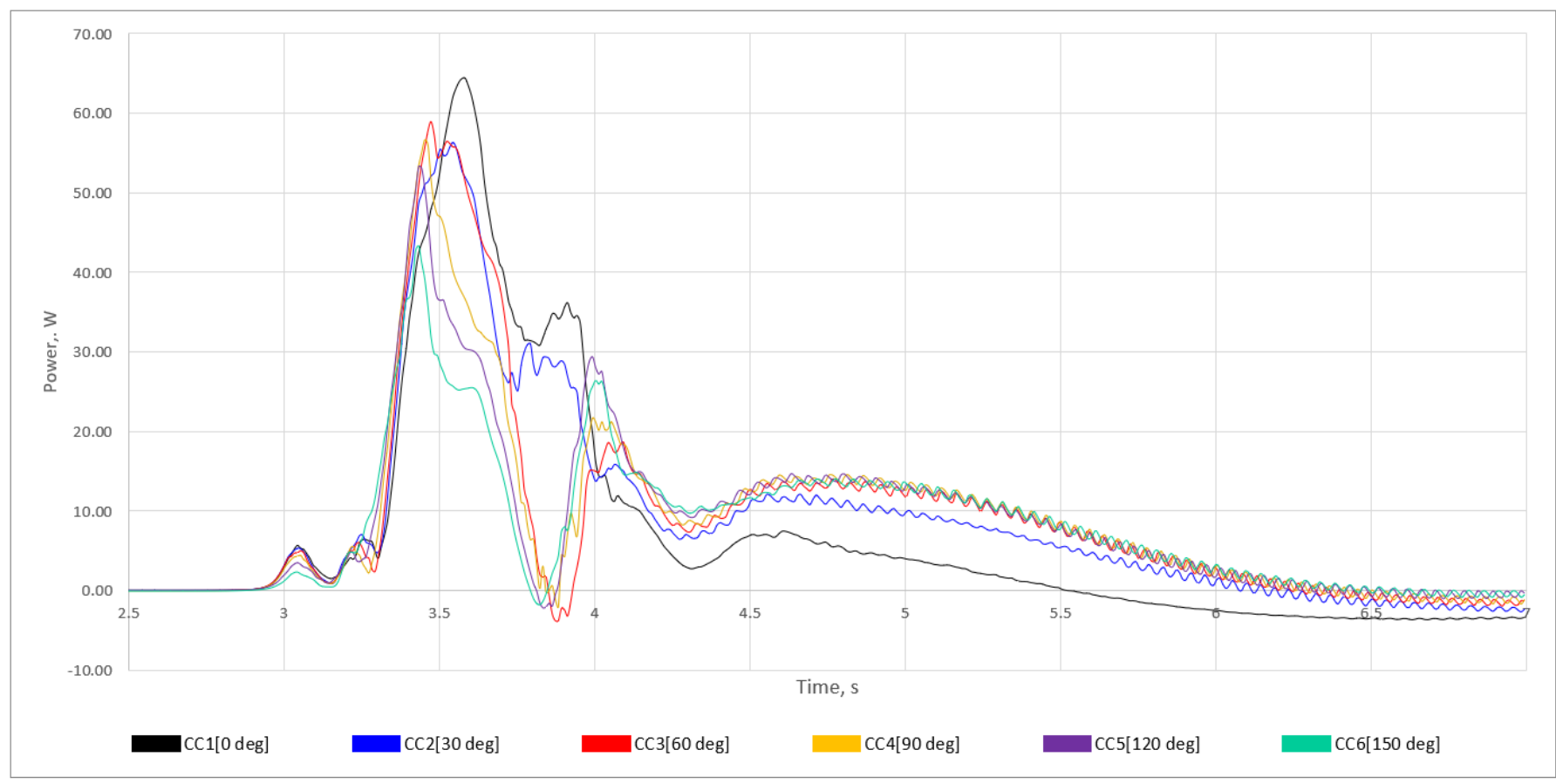

Figure 18. Instantaneous power of VAWT from Car Cases 1 to 6 (CC1 to CC6).

The power curve of each car case in Figure 18 generally shows three main parts: (1) the first rise and fall of the curve which includes the occurrence of peak value, (2) the second rise and fall but with lower magnitude than the previous, and (3) the third rise and fall of the curve which has the lowest magnitude and extends wider until it reaches the almost flat curve in $t>6 \mathrm{~s}$. 
In part 1 , the curve rises until it reaches the peak value at near $t \approx 3.5 \mathrm{~s}$ and then falls. This relates to the passage of the portion of the rear wake that contains high-velocity magnitude as shown in the contours in Figure 19. Upon inspection, all car cases have almost the same rear wake structure since it is only the windshield arclength that is varying between cases. The velocity contours captured at $t=3.5 \mathrm{~s}$ show that the portion of rear wake which has high velocity magnitude ends at this instance which explains why the curves fall afterward. It can be noticed that the Car Case 1, which has no windshield, gives the highest peak power. This is because the direction of the wind at this instance (near $t \approx 3.5 \mathrm{~s}$ ) is upward which makes the windshield ineffective in blocking the incoming wind that hits against the VAWT rotation and instead, creates stagnation in the area near the inner surface of the windshield. This stagnation causes the reduction of wind velocity from the center of the VAWT to the portion occupied by the windshield, this can be seen in the velocity contour and by comparing the length of lines of the velocity vector in Figure 20, where longer line represents higher magnitude. The Car Case 6 which has the longest arc length of 150 degrees, creates the largest flow stagnation, hence, this case has the lowest peak value and the lowest energy captured within the duration of part 1.

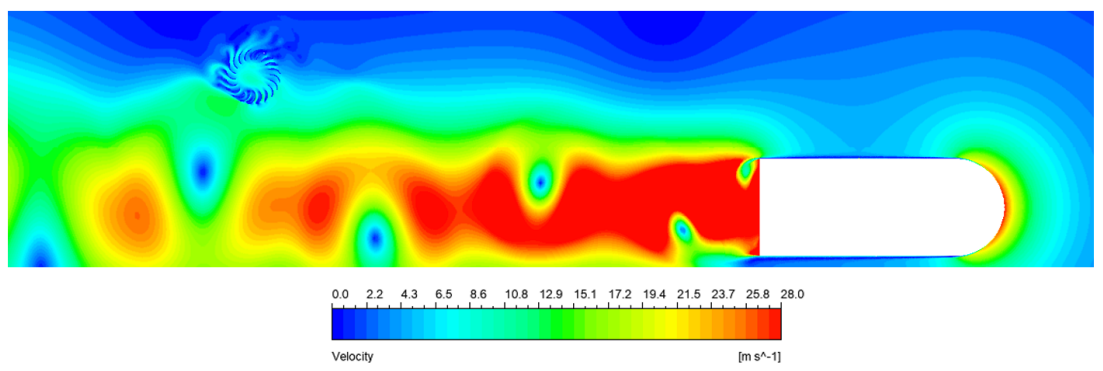

Figure 19. Velocity contours of each car case showing the rear wake taken at $t=3.5 \mathrm{~s}$.

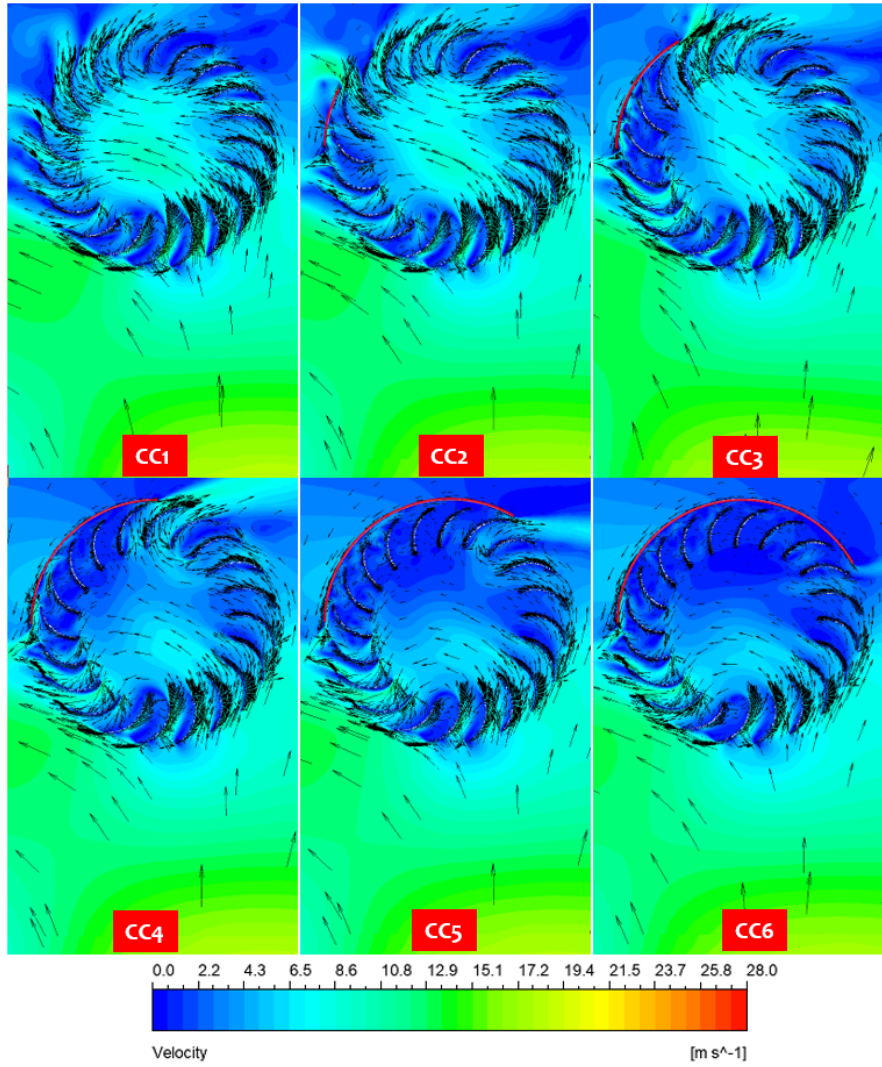

Figure 20. Velocity contours and vectors of each car case in the rotor zone taken at $t=3.5 \mathrm{~s}$. 
In the transition from part 1 to part 2 , which is the minima located at around $t \approx 3.8 \mathrm{~s}$, the curves of car cases with windshield fall deeper compared to that in Car Case 1. This effect is due to the wind direction pointing downward at this instance, as shown in Figure 21. For this wind direction, the chosen position of the windshield worsens the performance of VAWT since it blocks the incoming wind that supposedly pushes the blades to rotate and allows the incoming wind that hits the blades against its rotation. In the Car Case 2, the windshield is too short to totally block the wind that contributes to the positive torque, that is why the curve does not fall as deep as the other cases with the windshield.

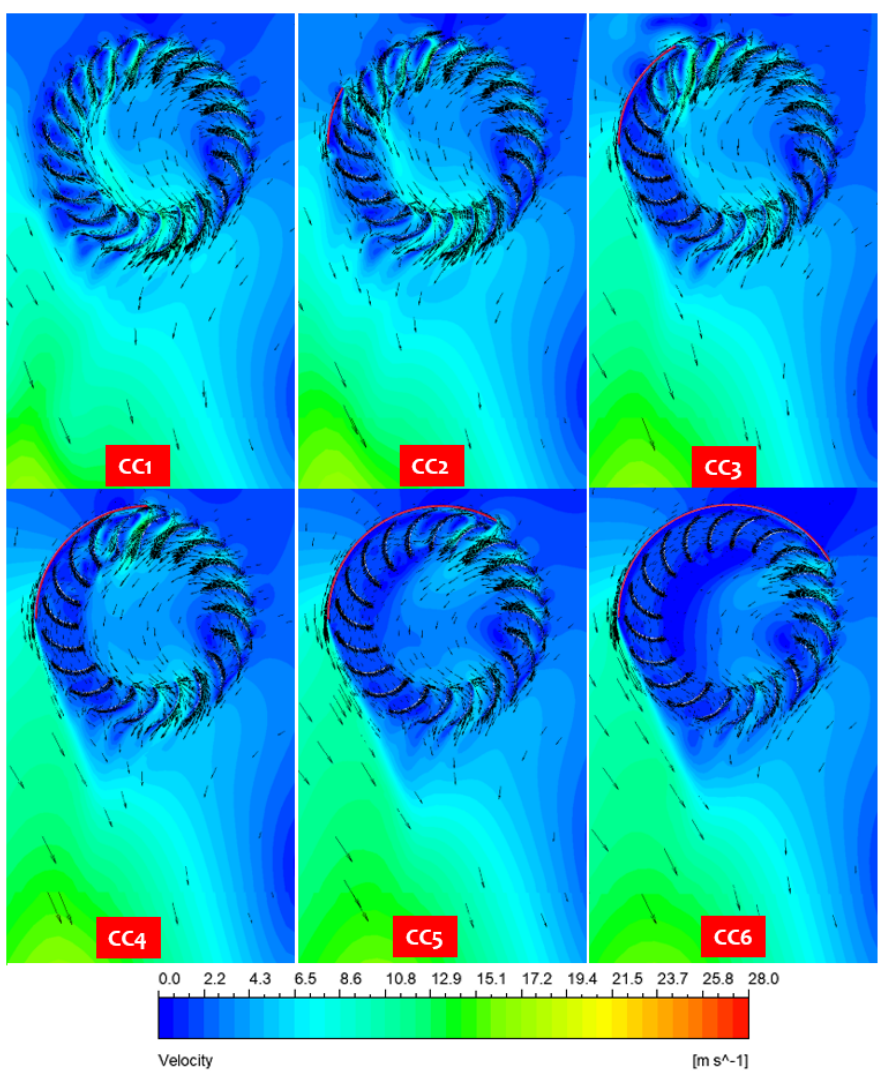

Figure 21. Velocity contours and vectors of each car case in the rotor zone taken at $t=3.8 \mathrm{~s}$.

From the minima, the curve starts to rise again and produce a smaller peak which is due to the apparent dissipation of vortices in this period. To visually show this, the wind direction on the period $t=4 \mathrm{~s}$ to $t=4.3 \mathrm{~s}$ for Car Case 3 is shown in Figure 22. The slightly varying wind direction is observed until near $t=5 \mathrm{~s}$ and eventually flatten to an almost horizontal direction beyond this instant. This direction is where the windshield gives an advantage. The velocity contour and vector for each car case in Figure 23 show the effectiveness of the windshield in blocking the wind hitting against the rotation. Looking back at the curve in part 3, the Car Case 2 gives the worst performance among cases with windshield, in the entire duration of part 3 of the curve. The reason is the windshield arc length in Car Case 2 being the shortest among other cases is not effective enough on blocking the wind hitting against the VAWT rotation, therefore, the increase in power from no windshield case is relatively low.

By taking the energy produced by one pass of the vehicle, which is represented by the area under the instantaneous power curve, gives the overall comparison of the effectiveness of windshield with varying arc length for car cases. The numerical integration is applied with negative values excluded, and the results of the computation are summarized in Table 3. It shows that having the windshield with a 60-degree arc length (Car Case 3) gives the highest increase in energy which is a $16.14 \%$ increase from no the windshield case. 


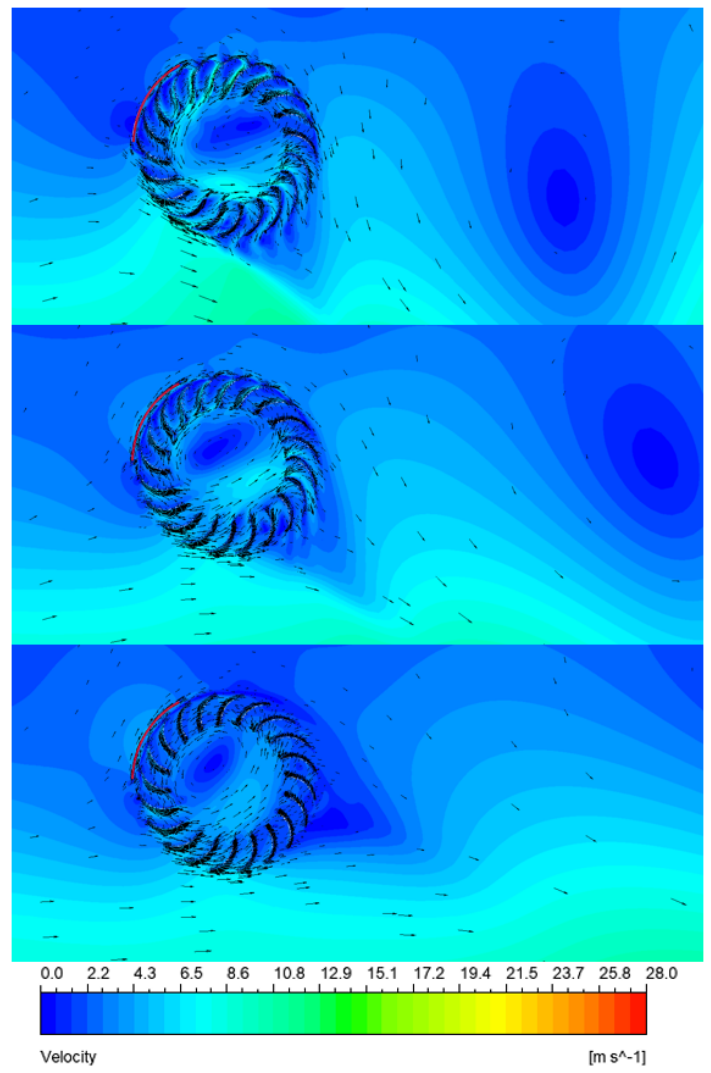

Figure 22. Velocity contours and vectors of Car Case 3 in the rotor zone taken at $t=4 \mathrm{~s}, t=4.1 \mathrm{~s}$ and $t=4.3 \mathrm{~s}$.

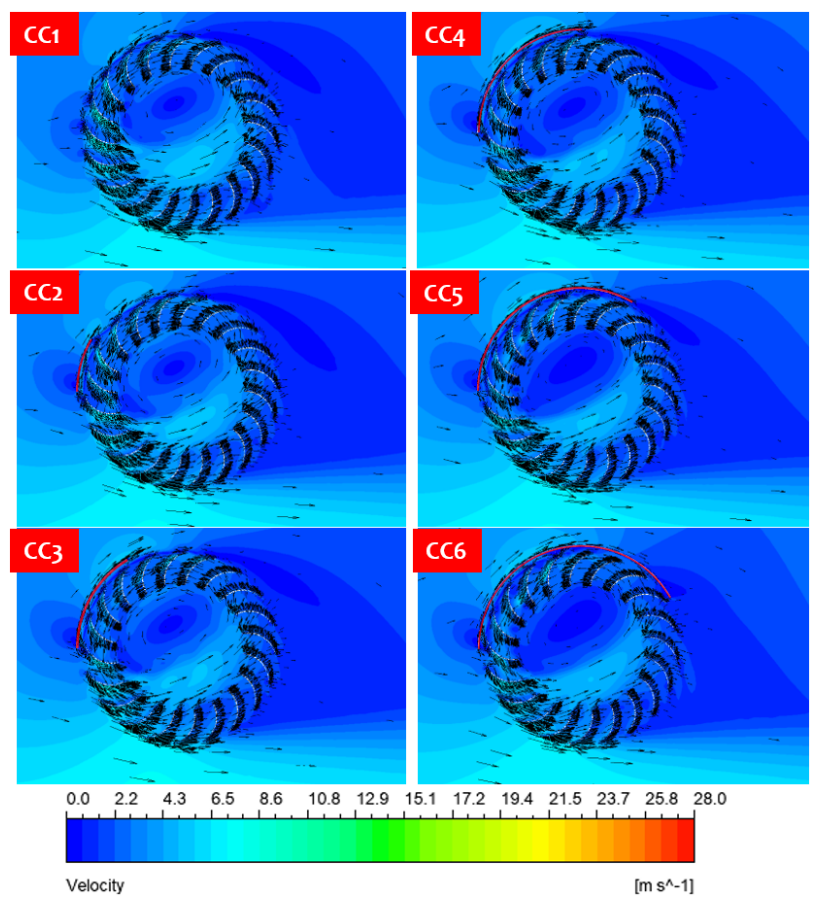

Figure 23. Velocity contours and vectors for Car Cases in the rotor zone taken at $t=5 \mathrm{~s}$. 
Table 3. Energy produced by VAWT in one pass of car for each case.

\begin{tabular}{cccc}
\hline Car Case & Arc Length, deg & Energy, Watt-Sec & \% Increase \\
\hline CC1 & 0 & 35.68 & 0.00 \\
CC2 & 30 & 41.42 & 16.08 \\
CC3 & 60 & 41.44 & 16.14 \\
CC4 & 90 & 40.63 & 13.88 \\
CC5 & 120 & 39.23 & 9.95 \\
CC6 & 150 & 36.61 & 2.62 \\
\hline
\end{tabular}

The same is done in the bus case and the resulting power curves are shown in Figure 24 followed by Table 4 which shows the energy produced for each bus case.

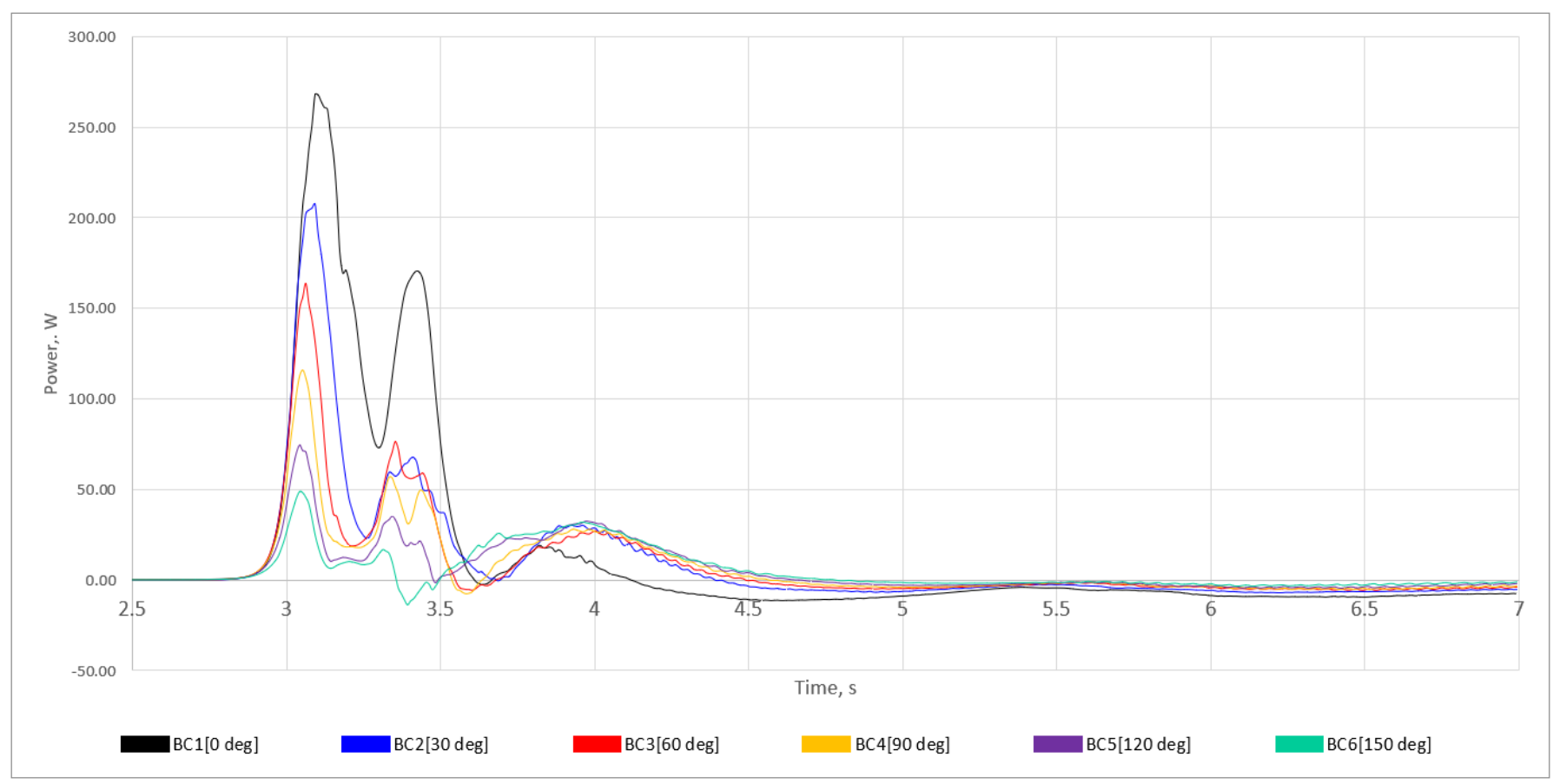

Figure 24. Instantaneous power of VAWT from Bus Cases 1 to 6 (BC1 to BC6).

Table 4. Energy produced by VAWT in one pass of bus for each case.

\begin{tabular}{cccc}
\hline Bus Case & Arc Length, deg & Energy, Watt-Sec & \% Increase \\
\hline BC1 & 0 & 87.96 & 0.00 \\
BC2 & 30 & 76.79 & -12.69 \\
BC3 & 60 & 46.19 & -47.48 \\
BC4 & 90 & 50.46 & -42.63 \\
BC5 & 120 & 39.32 & -55.30 \\
BC6 & 150 & 30.99 & -64.77 \\
\hline
\end{tabular}

Applying the same technique in getting the weighted-average wind direction in Section 3.2 to the bus case results in a weighted-average wind direction of 15.7 degrees from positive $\mathrm{x}$-axis.

As opposite to car cases, there is no improvement given by any of the bus cases with windshield and instead lowers the energy produced in the VAWT. Notice in Figure 25 that unlike in the car case, the wind direction in bus case remains alternating throughout until $t=7 \mathrm{~s}$. Thus, a stationary windshield that is effective to a particular wind direction did not contribute to the improvement of VAWT performance in bus case. The wind behavior is because the bus, which is wider and has lesser curvature at the front, produces larger 
vortices that take longer time to dissipate. This can visually see by comparing the velocity vector of bus wake in Figure 15 and that of car wake in Figure 13. The existence of slow dissipating vortices in the bus case also prohibits the VAWT to effectively harness the energy in the rear wake as supported by the instantaneous power curve that shows the curve being flat at $t>4.5 \mathrm{~s}$ while the car case is not.

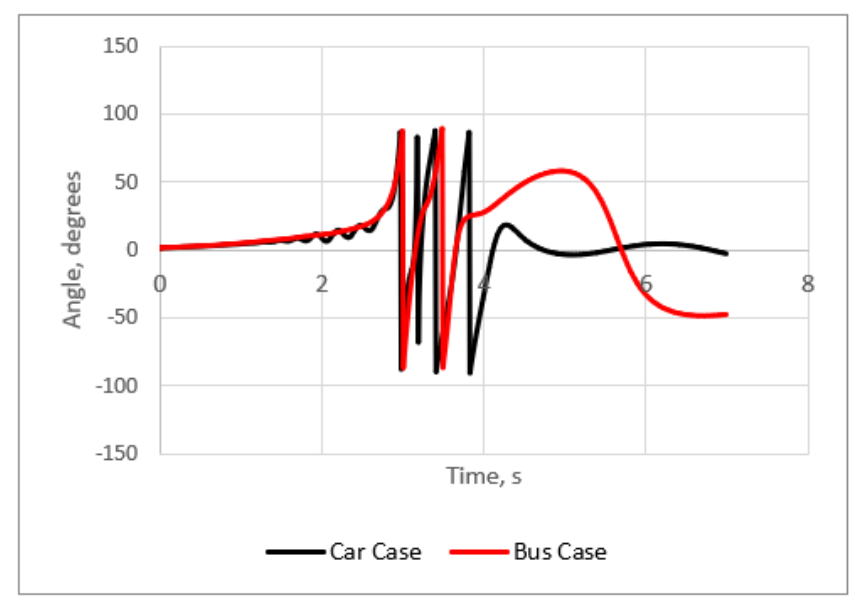

(a)

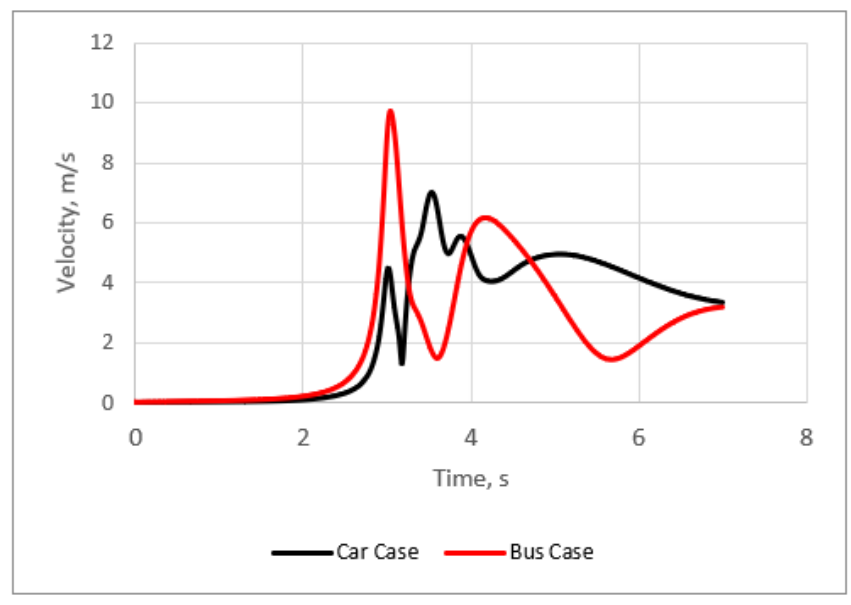

(b)

Figure 25. (a) Instantaneous wind direction at the rotor zone for car and bus models; (b) Instantaneous wind magnitude at the rotor zone for car and bus models.

\section{Conclusions}

The vehicle wake structure dictates the behavior of the power curve as proven by the part-by-part comparison of the curve against the corresponding velocity vectors and velocity contours. The installation of windshield affects the VAWT performance positively in the region where the vortices, created by the flow separation in vehicle walls, are already dissipated and gives negative effect when otherwise. The results show that the windshield provides an increase in the energy captured by the VAWT by up to $16.14 \%$ when the car model is used. In contrast, windshield in all the simulation cases using the bus model gives negative effect to VAWT performance where the worst case yields $-64.77 \%$. These findings suggest that the windshield is good when the VAWT is located in the road shoulder adjacent to the overtaking lane where large vehicles like buses are prohibited.

Author Contributions: Conceptualization, I.J.T.M.; methodology, I.J.T.M. and B.E.A.; software, I.J.T.M. and B.E.A.; validation, I.J.T.M.; formal analysis, I.J.T.M., L.A.M.D. and B.E.A.; resources, B.E.A.; writing—original draft preparation, I.J.T.M. and B.E.A.; writing-review and editing, I.J.T.M., L.A.M.D. and B.E.A.; visualization, I.J.T.M., L.A.M.D. and B.E.A.; supervision, B.E.A.; funding acquisition, L.A.M.D. All authors have read and agreed to the published version of the manuscript.

Funding: The APC was funded by Department of Science and Technology (DOST) Engineering Research and Development for Technology (ERDT) Program.

Acknowledgments: The authors would like to thank the Department of Science and Technology (DOST) Engineering Research and Development for Technology (ERDT) Program for the scholarship provided to the lead author of this manuscript for his graduate studies.

Conflicts of Interest: The authors declare no conflict of interest.

\section{References}

1. Global Energy Transformation: A Roadmap to 2050; IRENA (2019), International Renewable Energy Agency: Abu Dhabi, United Arab Emirates, 2019.

2. Gielen, D.; Boshell, F.; Saygin, D.; Bazilian, M.D.; Wagner, N.; Gorini, R. The Role of Renewable Energy in the Global Energy Transformation. Energy Strateg. Rev. 2019, 24, 38-50. [CrossRef] 
3. Al-Aqel, A.A.; Lim, B.K.; Noor, E.E.M.; Yap, T.C.; Alkaff, S.A. Potentiality of Small Wind Turbines along Highway in Malaysia. In Proceedings of the 2016 International Conference on Robotics, Automation and Sciences (ICORAS), Melaka, Malaysia, 5-6 November 2016; IEEE: New York, NY, USA, 2016; pp. 1-6. [CrossRef]

4. Bani-Hani, E.H.; Sedaghat, A.; Al-Shemmary, M.; Hussain, A.; Alshaieb, A.; Kakoli, H. Feasibility of Highway Energy Harvesting Using a Vertical Axis Wind Turbine. Energy Eng. 2018, 115, 61-74. [CrossRef]

5. Basilio, M.A.; Bernardo, J.M.; Cuya, J.M.; Luzano, J.L.; Chua, E.E.; Cudia, F.V.; Pacis, M.C. Harnessing of Electrical Energy Through Vehicular Air Drag on Highways For Lighting Load Applications. In Proceedings of the 2019 IEEE 11th International Conference on Humanoid, Nanotechnology, Information Technology, Communication and Control, Environment, and Management (HNICEM), Laoag, Philippines, 29 November-1 December 2019; IEEE: New York, NY, USA, 2019; pp. 1-5. [CrossRef]

6. Tian, W.; Song, B.; Mao, Z. Numerical Investigation of Wind Turbines and Turbine Arrays on Highways. Renew. Energy 2020, 147, 384-398. [CrossRef]

7. Tian, W.; Mao, Z.; Li, Y. Numerical Simulations of a VAWT in the Wake of a Moving Car. Energies 2017, 10, 478. [CrossRef]

8. Tian, W.; Mao, Z.; An, X.; Zhang, B.; Wen, H. Numerical Study of Energy Recovery from the Wakes of Moving Vehicles on Highways by Using a Vertical Axis Wind Turbine. Energy 2017, 141, 715-728. [CrossRef]

9. Tartuferi, M.; D'Alessandro, V.; Montelpare, S.; Ricci, R. Enhancement of Savonius Wind Rotor Aerodynamic Performance: A Computational Study of New Blade Shapes and Curtain Systems. Energy 2015, 79, 371-384. [CrossRef]

10. Burlando, M.; Ricci, A.; Freda, A.; Repetto, M.P. Numerical and Experimental Methods to Investigate the Behaviour of VerticalAxis Wind Turbines with Stators. J. Wind. Eng. Ind. Aerodyn. 2015, 144, 125-133. [CrossRef]

11. Wenlong, T.; Baowei, S.; Zhaoyong, M. A Numerical Study on the Improvement of the Performance of a Banki Wind Turbine. J. Wind. Eng. 2014, 38, 109-116. [CrossRef]

12. Nobile, R.; Vahdati, M.; Barlow, J.F.; Mewburn-Crook, A. Unsteady Flow Simulation of a Vertical Axis Augmented Wind Turbine: A Two-Dimensional Study. J. Wind Eng. Ind. 2014, 125, 168-179. [CrossRef]

13. Kim, D.; Gharib, M. Efficiency Improvement of Straight-Bladed Vertical-Axis Wind Turbines with an Upstream Deflector. J. Wind Eng. Ind. 2013, 115, 48-52. [CrossRef]

14. Chong, W.T.; Fazlizan, A.; Poh, S.C.; Pan, K.C.; Hew, W.P.; Hsiao, F.B. The Design, Simulation and Testing of an Urban Vertical Axis Wind Turbine with the Omni-Direction-Guide-Vane. Appl. Energy 2013, 112, 601-609. [CrossRef]

15. Chong, W.T.; Muzammil, W.K.; Ong, H.C.; Sopian, K.; Gwani, M.; Fazlizan, A.; Poh, S.C. Performance Analysis of the Deflector Integrated Cross Axis Wind Turbine. Renew. Energy 2019, 138, 675-690. [CrossRef] 